\title{
La certificación de competencias laborales en el programa de estudio de la carrera de Energías Renovables de la Universidad Tecnológica de Tijuana
}

\section{The work competencies certification in an educative program of Renewables Energies career of the Universidad Tecnológica de Tijuana}

TEÓN-VEGA, Argelia †*, BALDERAS-LÓPEZ, Silvia María, EATON-GONZÁLEZ, Bernardino Ricardo y CARPINTEYRO-CHÁVEZ, Lina Mariana

Universidad Tecnológica de Tijuana

ID $1^{\mathrm{er}}$ Autor: Argelia, Teón-Vega / ORC ID: 0000-0003-2274-6367, CVU CONACYT ID: 412627

ID $1^{\text {er }}$ Coautor: Silvia María, Balderas-López / ORC ID: 0000-0003-0434-5893, CVU CONACYT ID: 273260

ID $2^{\text {do }}$ Coautor: Bernardino Ricardo, Eaton González / ORC ID: 0000-0003-4528-4740, CVU CONACYT ID: 94473

ID $3^{\text {er }}$ Coautor: Lina Mariana, Carpinteyro-Chávez / ORC ID: 0000-0002-3452-6065, CVU CONACYT ID: 854251

DOI: $10.35429 / J U M .2019 .10 .3 .11 .19$

Recibido 19 de Octubre, 2019; Aceptado 03 de Diciembre, 2019

\begin{abstract}
Resumen
En los últimos cuatro años, la Universidad Tecnológica de Tijuana (UTT) ha implementado un programa de certificación de competencias laborales con los alumnos de la carrera de energías renovables, para validar sus habilidades en la instalación de sistemas fotovoltaicos interconectados (SFVI) y consolidar su formación técnica. Objetivo. El propósito de este proyecto es analizar la implementación del estándar de competencia laboral "EC0586.01 Instalación de sistemas fotovoltaicos en residencia, comercio e industria" en el programa educativo (PE) de la carrera de Energías Renovables de la UTT. Metodología. El proyecto se llevó a cabo en cuatro etapas: 1) alineación desde las materias del PE; 2) proceso de certificación; 3) resultados del proceso de evaluación e impresiones sobre el tema; 4) análisis de los resultados. Contribución. Este trabajo de investigación permitió conocer la pertinencia que tiene la implementación de un estándar de competencia laboral tanto en el PE de la UTT como en el mercado laboral de la carrera de Energías Renovables.
\end{abstract}

Certificación de competencias laborales, Instalación de SFVI, Programa educativo

\begin{abstract}
For the last four years, Tijuana's Technological University (UTT) has planned a workforce competence certification program with students from the renewables energies study program. The main goal is to validate their abilities on photovoltaic (pv) systems connected to the electrical grid and to consolidated their technician formation. The purpose of this study is to analyse impact of the implementation of the workforce competence standard "EC0586.01 Instalación de sistemas fotovoltaicos en residencia, comercio e industria" (Photovoltaic systems's installation in residence, commerce and industry) in educative program (EP) of the renewables energies career of the university. Methodology. The project was carried in four stages: 1) alignment of school subjects of EP; 2) certification process; 3 ) Results of the evaluation process and exchanges on the subject; 4) Analysis of the results reveals. Contribution. This investigation project was an opportunity to learn the relevance of the implementation of work competencies standard in the EP of UTT as in the case of work field of renewable energy career.
\end{abstract}

Workforce competence certification, Photovoltaic systems connected to the electrical grid, Educative program

Citación: TEÓN-VEGA, Argelia, BALDERAS-LÓPEZ, Silvia María, EATON-GONZÁLEZ, Bernardino Ricardo y CARPINTEYRO-CHÁVEZ, Lina Mariana. La certificación de competencias laborales en el programa de estudio de la carrera de Energías Renovables de la Universidad Tecnológica de Tijuana. Revista de Gestión Universitaria. 2019 3-10: 11-19

\footnotetext{
* Correspondencia del Autor (correo electrónico: argelia.teon@uttijuana.edu.mx)

$\uparrow$ Investigador contribuyendo como primer autor.
} 


\section{Introducción}

La educación y las competencias constituyen la base sobre la que México debe construir su crecimiento y prosperidad futura (OECD, 2017). Actualmente, en el sistema educativo escolarizado, se entiende que "el estudiante debe desarrollar una actitud que le permita cuestionar, analizar, reflexionar y actuar eficazmente sobre la sociedad en la que vive para mejorarla" (Jiménez, Hernández y Alfonso, 2013).

De acuerdo a Andrade (2008) y Chong y Castañeda (2013), la educación superior es un factor clave para el desarrollo de competencias, así como conocimientos técnicos, profesionales y disciplinares específicos en los estudiantes (Mertens, 2000), además de competencias transversales que les cualifican para una variedad de ocupaciones laborales (OECD, 2019).

En México, la implementación de los modelos de educación basados en competencias inició a mediados de los años noventa (Mertens, 1996), ya que permite garantizar tanto la formación de personal calificado que sustente nuevas formas de producción (Rojas, s.f.) como consolidar sistemas nacionales de elaboración, formación y certificación de competencias (Ruiz de Vargas, Jaraba y Romero, 2005).

Y es a través del Consejo de Normalización de Competencias Laborales (CONOCER) que se estableció un sistema de certificación de la capacidad o "competencia laboral" de los trabajadores con base en estándares de competencia (CONOCER, 2014).

$\mathrm{Al}$ evaluar una competencia laboral, se está midiendo el "saber hacer" de una persona (Ricart, 2014), ya que ser competente significa que es "capaz de desempeñar una función laboral de manera eficaz y eficiente en diferentes contextos de trabajo con los mismos resultados" (INIFED, 2012).

Entre las finalidades de la institución universitaria se podría decir que, además de proporcionar una preparación técnica y profesional, también contribuye al desarrollo crítico y participativo, ya que no puede quedarse solo en el papel de transmitir saberes teóricos (Ugarte y Naval, 2009).
En los últimos cuatro años, la Universidad Tecnológica de Tijuana (UTT) ha implementado un programa de certificación de competencias laborales en los distintos programas de estudio (PE) que ofrece, teniendo un estándar de competencia específico para cada uno de ellos. Para los estudiantes de la carrera de Técnico Superior Universitario (TSU) en Energías Renovables (ER) área Energía Solar, el estándar de competencia alineado a su formación es el EC0586.01 Instalación de sistemas fotovoltaicos en residencia, comercio e industria (DOF, 2017), que es la versión actualizada del EC0586 publicado en junio de 2015 en el Diario Oficial de la Federación (DOF, 2015).

El programa de certificación con los estudiantes de TSU ER se inició en el año 2016, y su principal objetivo es validar sus habilidades en la instalación de sistemas fotovoltaicos interconectados (SFVI) y consolidar su formación técnica. Cabe mencionar que la Facultad de Ingeniería Mecánica y Eléctrica de la Universidad Autónoma de Nuevo León ya contaba con un Centro Evaluador de Competencias Laborales, el cual impulsó un programa de certificaciones que formaban parte del plan de estudios de la carrera, siendo ésta una opción con doble beneficio: créditos en su programa educativo y comprobante de competencia laboral (Lara, Banda y Castillo, 2017).

El propósito del presente proyecto fue determinar la pertinencia de la certificación del estándar EC0586.01 como parte de la formación técnica de los estudiantes del PE de TSU ER. El desarrollo se llevó a cabo en cuatro etapas: 1) alineación desde las materias del plan de estudios; 2) proceso de certificación; 3) resultados del proceso de evaluación e impresiones sobre el tema; 4) análisis de los resultados.

Los resultados de la implementación del proceso de certificación del estándar de competencia laboral "EC0586.01 Instalación de sistemas fotovoltaicos en residencia, comercio e industria", permitieron conocer la forma en que la certificación contribuye al fortalecimiento del PE para complementar la formación técnica de los estudiantes.

Este trabajo muestra la experiencia que la UTT tuvo al incorporar la certificación de un estándar de competencias en el PE. 
Así es posible que otras instituciones educativas puedan valorar la inclusión de estándares en los PE, de acuerdo a su mapa curricular.

\section{Problemática}

La instalación de SFVI en residencia, comercio e industria, es un área del sector energético que se encuentra en crecimiento. El proceso de instalación de SFVI es exigente, laborioso y de mucha responsabilidad, y, en la mayoría de los casos, los técnicos instaladores no cuentan con una formación integral más allá del conocimiento empírico y la experiencia adquirida con el paso del tiempo. Debido a esto, se vuelve necesario contar con los recursos humanos que tengan las habilidades y conocimientos requeridos para realizar su trabajo con profesionalismo, además de buena actitud, hábitos y valores.

\section{Justificación}

Una alternativa para el desarrollo de habilidades y buenos hábitos en la instalación de SFVI es la implementación del estándar de competencia laboral "EC0586.01 Instalación de sistemas fotovoltaicos en residencia, comercio e industria" en el PE de la carrera de Energías Renovables (CONOCER, 2017). De esta manera, los estudiantes pueden obtener un complemento a su formación técnica, además de una certificación con valor curricular.

\section{Metodología}

Las etapas en las cuales se desarrolló el proyecto se muestran en la figura 1.

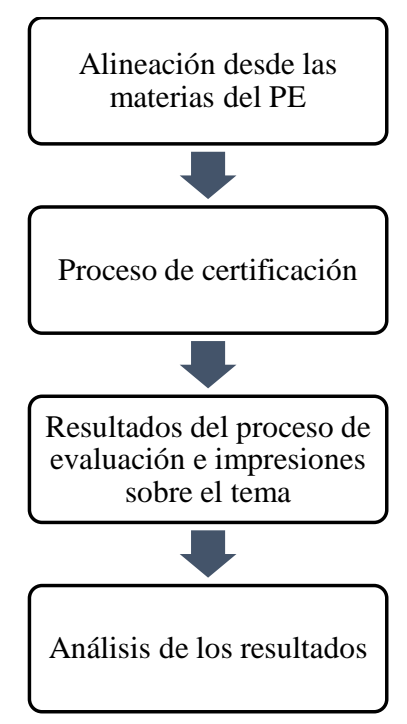

Figura 1 Diagrama de las etapas del proyecto Fuente: Elaboración Propia

\section{Alineación desde las materias del PE}

Se hizo una exploración del mapa curricular del PE de T.S.U. en Energías Renovables área Energía solar con la finalidad de determinar cuáles eran las materias desde las que se podía alinear los desempeños del estándar de competencia laboral y, mediante la revisión de las cartas descriptivas, desarrollar las prácticas pertinentes para que los estudiantes fueran adquiriendo las habilidades y conocimientos que les permitiera certificarse como instaladores de SFVI.

\section{Proceso de certificación}

\section{Preparación}

Como parte de su preparación para el proceso de certificación, los estudiantes realizaron prácticas de instalación durante el cuatrimestre previo a dicho proceso.

Se formaron equipos de trabajo de dos personas, y cada equipo estaba obligado a realizar al menos una práctica extra clase de instalación por semana, de manera que, al momento de ser evaluados, hubieran hecho de seis a ocho instalaciones.

\section{Evaluación}

La certificación se realiza al término del quinto cuatrimestre, que es cuando los estudiantes ya cubrieron los contenidos teóricos y prácticos necesarios para ser evaluados, y ésta consta de dos partes: la primera es un examen teórico, que evalúa conocimientos conceptuales, de normatividad y de aplicación, y la segunda consta de guías de observación para los desempeños prácticos.

\section{Resultados del proceso de evaluación e impresiones sobre el tema}

\section{Revisión de indicadores}

A la fecha, se cuenta con tres generaciones evaluadas en el EC0586 y EC0586.01. Se hizo una revisión de los indicadores por generación, para determinar los porcentajes de "Competente" y "Todavía no competente", así como de las fortalezas y áreas de oportunidad. 


\section{Aplicación de encuesta}

Se realizó un sondeo con los estudiantes, a través de una encuesta en línea, para conocer sus impresiones respecto a la certificación en su formación técnica y su repercusión en el área laboral.

En la tabla 1 se muestra el formato de la encuesta aplicada.

\begin{tabular}{|c|c|}
\hline \multicolumn{2}{|c|}{$\begin{array}{l}\text { La presente encuesta tiene como finalidad recabar información } \\
\text { sobre la certificación en el EC0586 y EC0586.01 Instalación } \\
\text { de sistemas fotovoltaicos interconectados en residencia, } \\
\text { comercio e industria, y sus ventajas en el ámbito laboral. }\end{array}$} \\
\hline \multicolumn{2}{|c|}{ 1. Mi género } \\
\hline \multicolumn{2}{|c|}{$\begin{array}{ll}\text { a) Masculino } & \text { b) Femenino }\end{array}$} \\
\hline \multicolumn{2}{|l|}{ 2. Mi estatus escolar es ... } \\
\hline \multicolumn{2}{|c|}{$\begin{array}{l}\text { b) Estudiante de ingeniería } \\
\text { en ER }\end{array}$} \\
\hline \multicolumn{2}{|c|}{$\begin{array}{l}\text { 3. ¿Cuál fue tu juicio de competencia de la certificación en el } \\
\text { EC0586/EC0586.01? }\end{array}$} \\
\hline Competente & Todavía no competente \\
\hline \multicolumn{2}{|c|}{$\begin{array}{l}\text { 4. ¿Trabajas en alguna empresa dedicada a la instalación de } \\
\text { sistemas fotovoltaicos? Si tu respuesta es "sí", menciona el } \\
\text { nombre de la empresa en la cual trabajas. }\end{array}$} \\
\hline a) Sí $\quad$ b) No & Nombre: \\
\hline \multicolumn{2}{|c|}{$\begin{array}{l}5 . . \text { ¿Consideras que estar certificado te da ventajas para tu } \\
\text { colocación como instalador de SFV? }\end{array}$} \\
\hline a) Sí ¿Por qué? & b) No ¿Por qué? \\
\hline \multicolumn{2}{|c|}{$\begin{array}{l}\text { 6. Con la certificación en el EC0586/EC0586.01 ... (puedes } \\
\text { indicar más de uno) }\end{array}$} \\
\hline \multirow{5}{*}{$\begin{array}{l}\text { a) se complementó mi } \\
\text { formación técnica } \\
\text { c) obtuve experiencia } \\
\text { como instalador de SFV } \\
\text { e) aprendí buenos hábitos } \\
\text { de seguridad } \\
\text { g) tuve preferencia sobre } \\
\text { otros candidatos } \\
\text { i) Otro: }\end{array}$} & $\begin{array}{l}\text { b) se puso a prueba mis } \\
\text { conocimientos }\end{array}$ \\
\hline & $\begin{array}{l}\text { d) adquirí confianza como } \\
\text { instalador de SFV }\end{array}$ \\
\hline & $\begin{array}{l}\text { f) aprendí a trabajar en } \\
\text { equipo }\end{array}$ \\
\hline & $\begin{array}{l}\text { h) me he cotizado mejor en el } \\
\text { ámbito laboral }\end{array}$ \\
\hline & \\
\hline \multicolumn{2}{|c|}{ 7. ¿Recomendarías esta certificación? } \\
\hline a) Sí ¿Por qué? & b) No ¿Por qué? \\
\hline
\end{tabular}

Tabla 1. Formato de la encuesta aplicada.

Fuente: Elaboración Propia

\section{Análisis de los resultados}

Se procedió a realizar un análisis de los indicadores por generación y de las áreas de oportunidad detectadas, de manera que se pudiera proponer acciones de mejora.

A su vez, con el análisis del sondeo se pretendía obtener información de primera mano sobre los beneficios y oportunidades laborales obtenidos con la certificación.

\section{Resultados}

\section{Materias del PE alineadas al estándar de competencia laboral}

Al hacer la revisión del mapa curricular, se encontró que ocho de las materias del PE se podían alinear al estándar de competencia laboral, y a su vez se revisaron las cartas descriptivas. En la tabla 2 se muestran dichas materias, así como los temas y prácticas relacionados con el estándar.

\begin{tabular}{|c|c|c|}
\hline Cuatrimestre & Materias & Temas \\
\hline $1^{\circ}$ & $\begin{array}{l}\text { Electricidad y } \\
\text { magnetismo }\end{array}$ & $\begin{array}{l}\text { Conceptos de } \\
\text { electricidad, corriente } \\
\text { directa y corriente alterna } \\
\text { Medición de parámetros } \\
\text { eléctricos. }\end{array}$ \\
\hline $2^{\circ}$ & $\begin{array}{l}\text { Instalaciones } \\
\text { eléctricas }\end{array}$ & $\begin{array}{l}\text { Todos los contenidos. } \\
\text { Elaboración } \\
\text { diagramas unifilares. }\end{array}$ \\
\hline $3^{\circ}$ & $\begin{array}{l}\text { Energías } \\
\text { renovables }\end{array}$ & $\begin{array}{l}\text { Incidencia de la radiación } \\
\text { solar. } \\
\text { Energía } \\
\text { fotovoltaica. } \\
\text { Prácticas de orientación, } \\
\text { determinación de horas y } \\
\text { días del año con mayor } \\
\text { incidencia solar. }\end{array}$ \\
\hline \multirow[b]{3}{*}{$4^{\circ}$} & $\begin{array}{l}\text { Estructura y } \\
\text { propiedades de } \\
\text { los materiales }\end{array}$ & \begin{tabular}{lcc}
$\begin{array}{l}\text { Definición } \\
\text { propiedades }\end{array}$ & de & las \\
materiales, & tipos \\
estructuras & metálicas \\
resistencia. & \multicolumn{2}{c}{} \\
\end{tabular} \\
\hline & $\begin{array}{l}\text { Dibujo } \\
\text { industrial }\end{array}$ & $\begin{array}{l}\text { Todos los contenidos. } \\
\text { Elaboración de planos en } \\
\text { AutoCAD. }\end{array}$ \\
\hline & $\begin{array}{l}\text { Estaciones } \\
\text { meteorológicas }\end{array}$ & $\begin{array}{l}\text { Concepto de insolación, } \\
\text { procedimientos de } \\
\text { medición, conversión de } \\
\text { unidades de radiación } \\
\text { solar. Características de } \\
\text { los instrumentos para } \\
\text { medición de radiación } \\
\text { solar. } \\
\text { Medición de radiación } \\
\text { solar. }\end{array}$ \\
\hline \multirow[b]{2}{*}{$5^{\circ}$} & $\begin{array}{l}\text { Celdas } \\
\text { fotovoltaicas }\end{array}$ & $\begin{array}{l}\text { Todos los contenidos. } \\
\text { Prácticas de instalación } \\
\text { de SFVI. }\end{array}$ \\
\hline & $\begin{array}{l}\text { Seguridad } \\
\text { industrial }\end{array}$ & $\begin{array}{l}\text { Fundamentos } \\
\text { seguridad e higiene } \\
\text { industrial, equipo de } \\
\text { protección personal, } \\
\text { riesgos y accidentes, } \\
\text { normas aplicables. }\end{array}$ \\
\hline
\end{tabular}

Tabla 2 Materias del PE alineadas al estándar de competencia

Fuente: Elaboración Propia con información de las cartas descriptivas del PE de TSU E.R

\section{Proceso de certificación}

\section{Prácticas realizadas}

La secuencia de prácticas realizadas se hizo conforme al orden mostrado en la tabla 2.

TEÓN-VEGA, Argelia, BALDERAS-LÓPEZ, Silvia María, EATONGONZÁLEZ, Bernardino Ricardo y CARPINTEYRO-CHÁVEZ, Lina Mariana. La certificación de competencias laborales en el programa de estudio de la carrera de Energías Renovables de la Universidad Tecnológica de Tijuana. Revista de Gestión Universitaria. 2019 


\begin{tabular}{|r|l|}
\hline \multicolumn{2}{|c|}{ \# } \\
\hline 1 & Usóctica \\
\hline 2 & Verificación de sitio para instalación \\
\hline 3 & $\begin{array}{l}\text { Orientación con brújula y puesta de taquetes para } \\
\text { anclaje }\end{array}$ \\
\hline 4 & $\begin{array}{l}\text { Levantamiento de estructura y fijación de } \\
\text { módulos FV }\end{array}$ \\
\hline 5 & Instalación de canalizaciones \\
\hline 6 & $\begin{array}{l}\text { Medición de parámetros eléctricos de los módulos } \\
\text { FV }\end{array}$ \\
\hline 7 & Conexión de componentes eléctricos \\
\hline 8 & Puesta en marcha del sistema \\
\hline
\end{tabular}

Tabla 3 Secuencia de prácticas de preparación Fuente: Elaboración Propia

En las figuras 2 y 3 se muestran imágenes de algunas de estas prácticas.

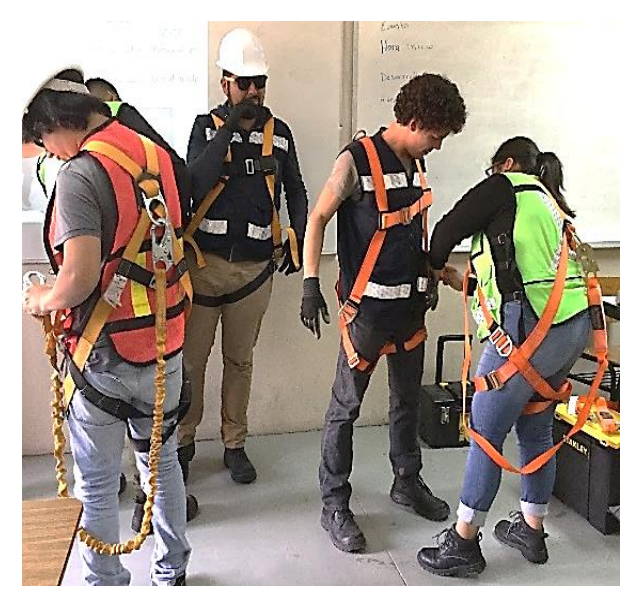

Figura 2 Uso del equipo de protección personal Fuente: Elaboración Propia

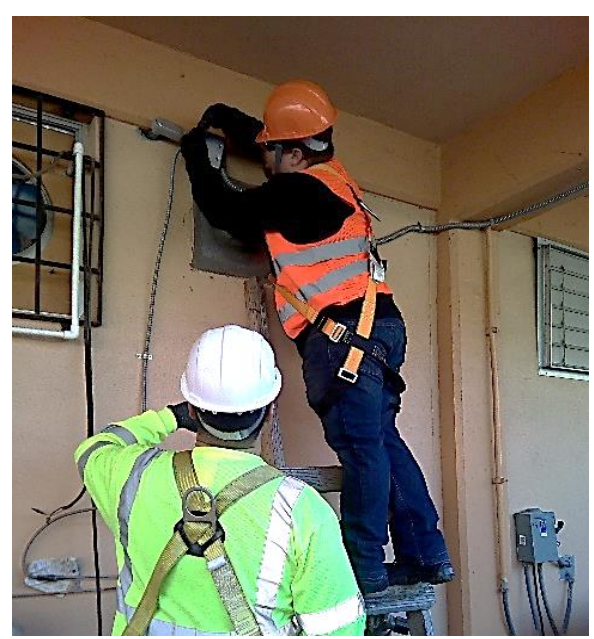

Figura 3 Instalación de canalizaciones Fuente: Elaboración Propia

\section{Evaluación}

La evaluación tiene una duración entre ocho y diez horas. En la secuencia fotográfica de las figuras 4 a 6 se muestran algunos de los procesos de evaluación realizados.

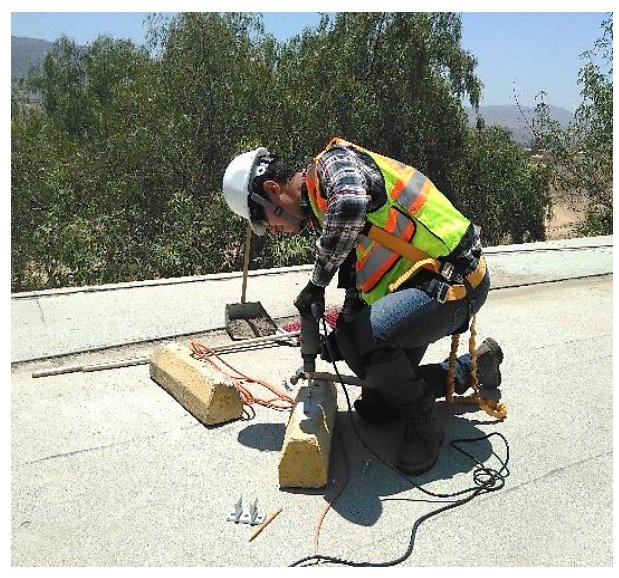

Figura 4 Preparación del anclaje para la estructura Fuente: Elaboración Propia

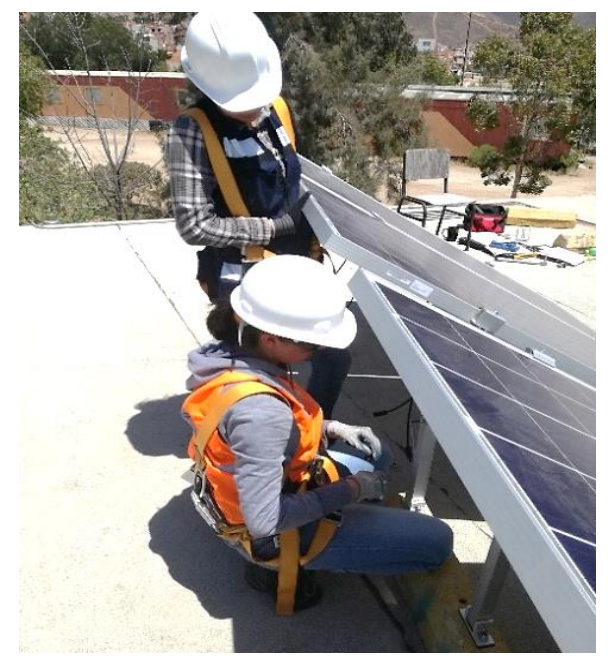

Figura 5 Fijación de módulos FV

Fuente: Elaboración Propia

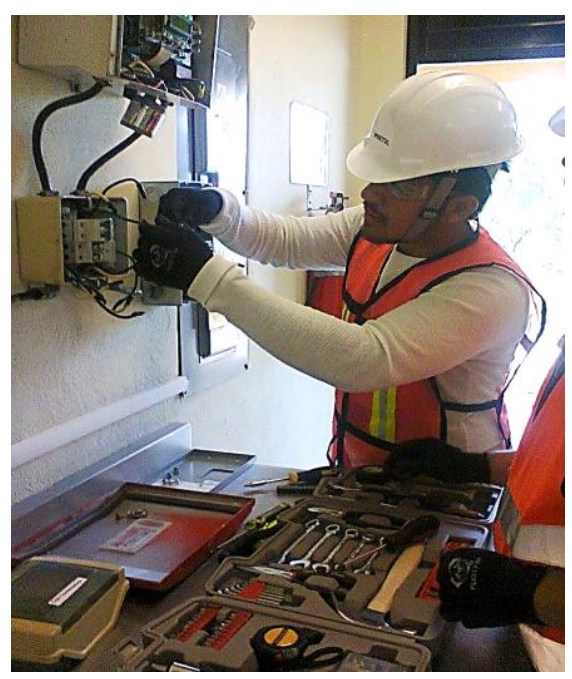

Figura 6 Conexión de componentes eléctricos Fuente: Elaboración Propia

\section{Resultados obtenidos}

\section{Indicadores de competencia}

Se revisaron los indicadores por generación para la determinación de porcentajes del nivel de competencia logrado. En la tabla 4 se resumen los indicadores por generación.

TEÓN-VEGA, Argelia, BALDERAS-LÓPEZ, Silvia María, EATONGONZÁLEZ, Bernardino Ricardo y CARPINTEYRO-CHÁVEZ, Lina Mariana. La certificación de competencias laborales en el programa de estudio de la carrera de Energías Renovables de la Universidad Tecnológica de Tijuana. Revista de Gestión Universitaria 2019 


\begin{tabular}{|c|r|r|r|}
\hline $\begin{array}{c}\text { Generación } \\
\text { de TSU }\end{array}$ & $\begin{array}{c}\text { Cantidad } \\
\text { de } \\
\text { alumnos } \\
\text { evaluados }\end{array}$ & \multicolumn{2}{|c|}{ Juicio de competencia } \\
\hline $2014-2016$ & 19 & 16 & $\begin{array}{r}\text { Todavía no } \\
\text { competente }\end{array}$ \\
\hline $2015-2017$ & 19 & 15 & 4 \\
\hline $2016-2018$ & 22 & 19 & 3 \\
\hline
\end{tabular}

Tabla 4 Indicadores de las certificaciones por generación Fuente: Elaboración Propia

Como fortalezas encontradas, tenemos:

- $\quad$ Uso del equipo de protección personal.

- $\quad$ Coordinación para trabajar en equipo.

- Buen manejo de los componentes a instalar y herramienta.

- Uso de la herramienta correcta para funciones específicas.

- Verificación de los elementos de seguridad en la instalación eléctrica.

- Interpretación de diagramas eléctricos y simbología.

Respecto a las áreas de oportunidad detectadas, se puede mencionar las siguientes:

- $\quad$ Dificultades para que la estructura y los módulos queden alineados.

- $\quad$ Falta de optimización de tiempo.

- Falta de habilidad para detectar fallos durante la instalación e interconexión.

- $\quad$ Elaboración de la carpeta de proyecto.

- Conceptos básicos de electricidad.

- Conocimiento de las características de los conductores.

\section{Resultados de la encuesta}

En la tabla 5 se muestran los resultados de la encuesta por pregunta.

\begin{tabular}{|l|l|}
\hline Pregunta 1: mi género & 24 \\
\hline Masculino & 9 \\
\hline Femenino & 18 \\
\hline Pregunta 2: mi estatus escolar \\
\hline Egresado & 15 \\
\hline Estudiante de ingeniería en ER & 0 \\
\hline Baja temporal & $\begin{array}{l}\text { Pregunta 3: ¿Cuál fue tu juicio de competencia de la } \\
\text { certificación en el EC0586/EC0586.01? }\end{array}$ \\
\hline Competente & 30 \\
\hline Todavía no competente & 3 \\
\hline $\begin{array}{l}\text { Pregunta 4: ¿Trabajas en alguna empresa dedicada } \\
\text { a la instalación de sistemas fotovoltaicos? }\end{array}$ \\
\hline Sí & 16 \\
\hline No & 17 \\
\hline
\end{tabular}
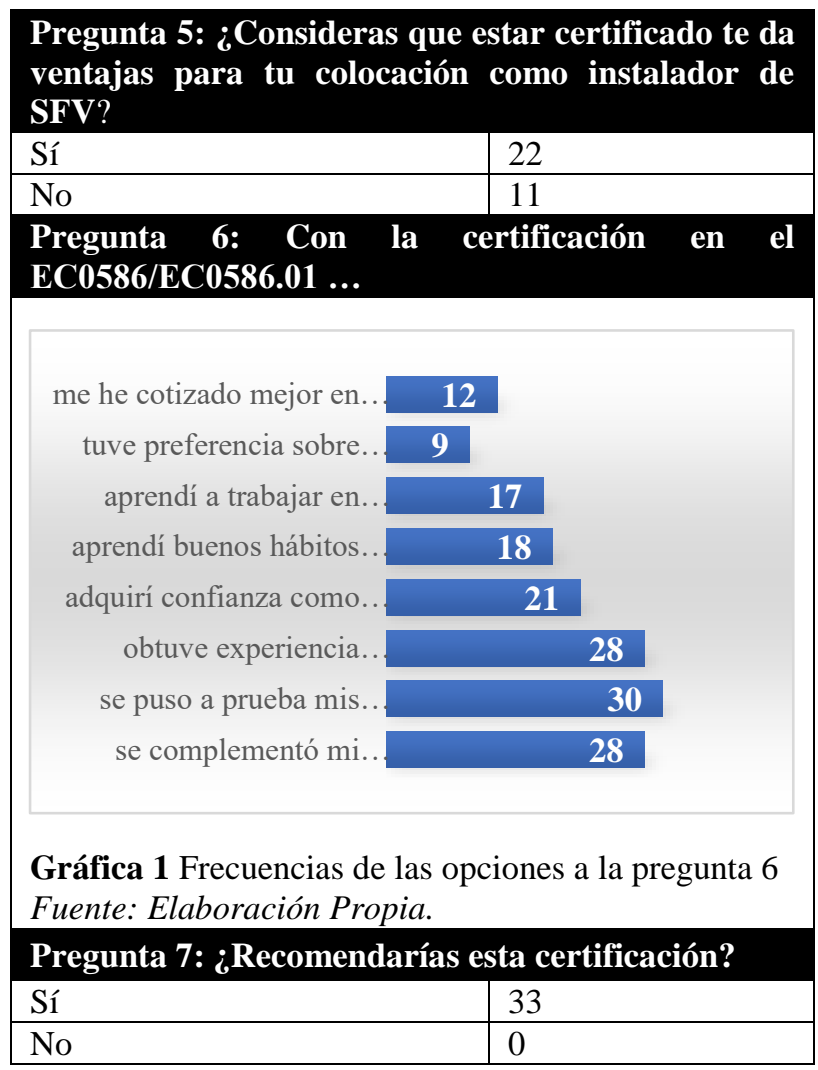

Tabla 5 Resultados de la encuesta

Fuente: Elaboración Propia

\section{Análisis de resultados}

\section{Indicadores y áreas de oportunidad}

A la fecha, se han evaluado 60 estudiantes, de los cuales 50 han resultado competentes, lo cual representa un $83 \%$ de competencia en lo que lleva implementada la certificación. La respuesta de los estudiantes respecto al proceso ha sido positiva y asumen de buen agrado todo lo referente a su preparación. Conforme van realizando las prácticas se nota el avance gradual en los desempeños, manejo de herramienta $\mathrm{y}$, sobre todo, la confianza en sí mismos. Sin embargo, aún quedan varios puntos en los cuales trabajar.

Respecto a las áreas de oportunidad detectadas, las dificultades en el alineamiento de la estructura y los módulos, la falta de optimización de tiempo y de habilidad para la detección de fallos, se debe mayormente a que aún no tienen la experiencia en campo que les permita un mayor dominio de esas destrezas. Referente a la carpeta de proyecto, a pesar de que se empieza a elaborar con anticipación, todavía no se ha logrado que ésta sea $100 \%$ satisfactoria, generalmente cubre los mínimos requeridos, siendo ésta la mayor área de oportunidad encontrada. 
Y en cuanto a los conceptos básicos de electricidad y conocimiento de características de los conductores, son temas que se cubren casi al inicio de su formación como TSU, y al momento de la certificación no los traen tan presentes. Se debe poner mayor énfasis desde las materias en las cuales se trabajan estos temas, además del repaso general previo a la evaluación.

\section{Encuesta aplicada}

La encuesta fue enviada a todos los estudiantes que han sido evaluados, de los cuales respondieron 33, lo que representa un $55 \%$ de este grupo de estudio. Del análisis realizado a los indicadores que arrojó el sondeo tenemos lo que se muestra en los siguientes puntos:

- $\quad$ Se perfila un $27 \%$ del género femenino (gráfica 2) entre los encuestados. Aunque gradualmente se incorporan más chicas al área de la instalación de SFV, todavía es un sector en el que predomina el género masculino.

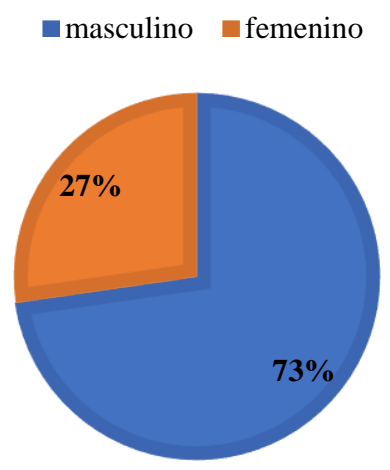

Gráfica 2 Género de los encuestados Fuente: Elaboración Propia

El $55 \%$ son egresados y el $45 \%$ aún se encuentran estudiando (gráfica 3 ).

— egresado $\square$ estudiante de ingeniería en ER

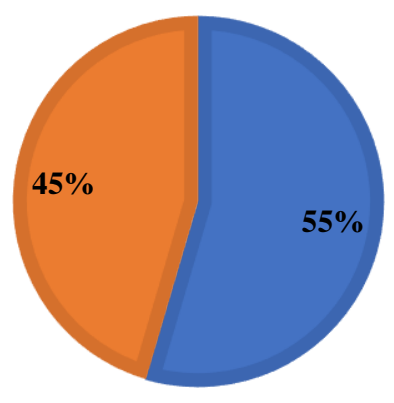

Gráfica 3 Estatus escolar

Fuente: Elaboración Propia
El 91\% obtuvo como juicio de competencia "competente" (gráfica 4).

competente $\quad$ todavía no competente

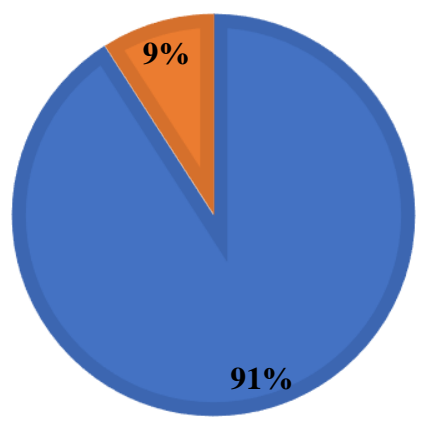

Gráfica 4 Juicio de competencia

Fuente: Elaboración Propia

- $\quad$ El 52\% no trabaja en empresas dedicadas a la instalación de SFV. En gran parte se debe a que un $45 \%$ de los encuestados aún se encuentra estudiando (gráfica 3).

De los encuestados que sí están trabajando en empresas dedicadas a la instalación de SFV, algunas de las mencionadas son:

\section{- Ecoglobe}

- Tecnoenergía Alternativa de México

- IPEC Industrial Área Solar

- Exel Solar

Y mención aparte para dos empresas creadas por egresados:

\section{- Mexsun \\ - $\quad$ FEM Instalaciones}

- $\quad$ El $67 \%$ considera que estar certificado sí les da ventajas en su colocación como instaladores de SFV.

Algunos de sus comentarios al respecto son:

- "Se adquiere mayor seguridad en tu desempeño".

"Te cotizas mejor en el medio".

$-$

- $\quad$ "Puedo ejercer el trabajo oficialmente, con los conocimientos adquiridos".

"Perfil más competente". 
- De los beneficios que ellos consideran que obtuvieron con la certificación, poner a prueba sus conocimientos es el que predomina con un $18 \%$, seguido de que fue un complemento a su formación técnica y obtener experiencia como instaladores con un $17 \%$. Solo el $6 \%$ considera que tuvo preferencia sobre otros candidatos (gráfica 5).

\footnotetext{
— se complementó mi formación técnica

- se puso a prueba mis conocimientos

- obtuve experiencia como instalador de SFV

adquirí confianza como instalador de SFV

—aprendí buenos hábitos de seguridad

- aprendí a trabajar en equipo

- tuve preferencia sobre otros candidatos

- me he cotizado mejor en el ámbito laboral
}

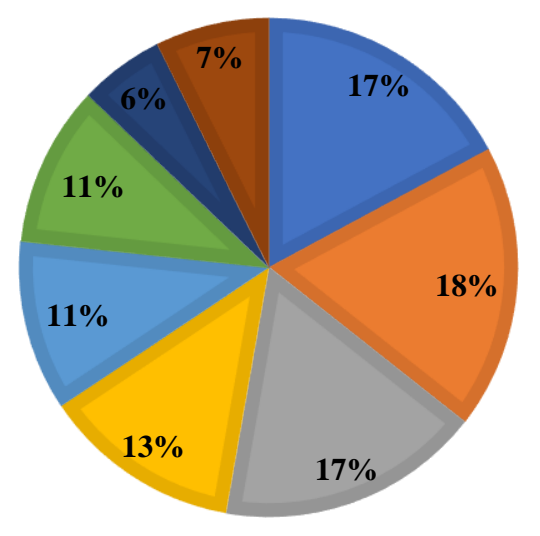

Gráfica 5 Beneficios obtenidos con la certificación. Fuente: Elaboración Propia

- $\quad$ El $100 \%$ de los encuestados recomienda la certificación.

Sus comentarios al respecto son:

- "Con esta certificación se ponen en práctica todos los conocimientos recibidos por parte de la universidad".

- $\quad$ "Ayuda al momento de solicitar empleo con relación a las instalaciones de sistemas fotovoltaicos".

- _Da mejor preparación laboral".

- "Te da seguridad, tiene valor curricular, y también te da cierta preferencia en el campo laboral".

\section{Agradecimiento}

Agradecemos a la Universidad Tecnológica de Tijuana por facilitar las condiciones para que se llevaran a cabo los procesos de certificación.
Al equipo de evaluadores encabezado por el M.C. Luis Eduardo Vargas Gurrola, por su apoyo y dedicación para que esto fuera posible.

\section{Conclusiones}

Una vez terminado el presente estudio se identifica que la certificación es pertinente al plan de estudios, ya que durante la primera etapa se identificó que los elementos del estándar están alineados con las cartas descriptivas de ocho materias del PE.

El $83 \%$ de los estudiantes, de las tres generaciones que se han certificado, resultaron competentes en la función evaluada. Las fortalezas que aporta el estándar van desde el manejo de los componentes a instalar y el uso de herramienta de acuerdo a la actividad.

El estudio hizo posible reconocer las áreas de oportunidad del desempeño de los estudiantes, como la falta de habilidad para detectar fallos durante la instalación e interconexión.

El sondeo permitió conocer la percepción que tienen los alumnos con respecto a la certificación. Entre los beneficios que se identificaron están poner a prueba los conocimientos y, consideran el estándar, como un complemento a su formación técnica.

\section{Referencias}

Andrade, R., (2008). El enfoque por competencias en educación. Ideas CONCYTEG. Año 3. No. 39.

Chong, M, y Castañeda, R., (2013) Sistema educativo en México: El modelo de competencias, de la industria a la educación. Sincronía, 4-6.

Consejo de Normalización de Competencias Laborales (CONOCER), (2014). Qué es el sistema nacional de competencias. Recuperado de http://www.conocer.gob.mx.

Consejo de Normalización de Competencias Laborales (CONOCER), (2017). Recuperado en https://conocer.gob.mx/acciones_programas/est andar-de-competencia/

Diario oficial de la federación, 2015, EC0586 Instalación de sistemas fotovoltaicos en residencia, comercio e industria.

TEÓN-VEGA, Argelia, BALDERAS-LÓPEZ, Silvia María, EATONGONZÁLEZ, Bernardino Ricardo y CARPINTEYRO-CHÁVEZ, Lina Mariana. La certificación de competencias laborales en el programa de estudio de la carrera de Energías Renovables de la Universidad Tecnológica de Tijuana. Revista de Gestión Universitaria. 2019 
Diario oficial de la federación, 2017, EC0586.01 Instalación de sistemas fotovoltaicos en residencia, comercio e industria.

Jiménez, Y., Hernández, J. y Alfonso, M., (2013) Competencias profesionales en la educación superior: justificación, evaluación y análisis. Innovación educativa. Volumen13. 4565.

Instituto nacional de infraestructura educativa, (2012) Evaluación para la certificación de competencias laborales, recuperado de https://www.gob.mx/inifed/acciones-yprogramas/evaluacion-para-la-certificacion-decompetencias-laborales

Lara, C., Banda, F. y Castillo, J. (2017). Certificaciones en competencias laborales en los programas educativos de ingeniería. ANFEI Digital, 1-7.

Mertens, L. (1996) Competencia laboral: sistemas, surgimiento y modelos, 50 .

Mertens, L. (2000) Formación basada en competencia laboral: situación actual y perspectivas

OECD (2017), OECD Skills Strategy Diagnostic Report: Mexico 2017, OECD Skills Studies, OECD Publishing, Paris. Recuperado de http://dx.doi.org/10.1787/9789264287679-en.

OECD (2019), Higher Education in Mexico: Labour Market Relevance and Outcomes, Higher Education, OECD Publishing, Paris, https://doi.org/10.1787/9789264309432-en.

Ricart, C. (2014). Construyendo un sistema de aprendizaje a lo largo de la vida en México. México: Banco Interamericano de Desarrollo

Rojas, I., (s.f.) La educación basada en normas de competencia (ERNe) como un nuevo modelo de formacion profesional en México. Universidad abierta UNAM.

Ruiz de Vargas, M., Jaraba, B. y Romero, L., (2005). Competencias laborales y la formación universitaria. Psicología del Caribe, 64-91.

Ugarte, C. y Naval, C., (2009) Desarrollo de competencias profesionales en la educación superior, recuperado de http://redie.uabc.mx/contenido/NumEsp2/conte nido-ugarte.html
TEÓN-VEGA, Argelia, BALDERAS-LÓPEZ, Silvia María, EATONGONZÁLEZ, Bernardino Ricardo y CARPINTEYRO-CHÁVEZ, Lina Mariana. La certificación de competencias laborales en el programa de estudio de la carrera de Energías Renovables de la Universidad Tecnológica de Tijuana. Revista de Gestión Universitaria. 2019 